\title{
Response to Letter to the Editor by Ernesto de Bernadis
}

\author{
Flavio Moroni Guido Mannaioni \\ Department of Neurofarba, University of Firenze, Florence, Italy
}

\section{Dear Editor,}

The target therapeutic plasma R-methadone concentration for the treatment of severe heroin users in the methadone maintenance program (MMT) described in Mannaioni et al. [1] was set at 80-250 ng/ $\mathrm{mL}$. The comment letter of de Bernardis suggests that by further increasing plasma R-methadone concentrations, a better therapeutic outcome could possibly be obtained. This suggestion is based on data published several years ago by Eap et al. [2].

When we selected the therapeutic window of $80-250 \mathrm{ng} / \mathrm{mL}$ of trough plasma Rmethadone levels, we were certainly aware of the content of Eap et al. [2, 3] papers, which included the information that $93 \%$ of the MMT patients having plasma concentrations above $250 \mathrm{ng} / \mathrm{mL}$ of R-methadone do not use illicit opioids [2]. Our main goal was not limited to reduce illicit drug use, but we aimed to improve the percentage of patients retained in treatment for 1 year and to improve their quality of life. To reach this goal, we first selected $100-250 \mathrm{ng} / \mathrm{mL}$ as a reasonable therapeutic plasma concentration of the active methadone enantiomer. The selection was based not only on our preliminary unpublished data but also on the careful evaluation of the content of the above-mentioned Eap et al. [2] publication (see the original protocol: Eudra C.T.2008-005028-10). In the first weeks of the trial, however, we realized that most patients in MMT having plasma $\mathrm{R}$-methadone concentration between 80 and $100 \mathrm{ng} / \mathrm{mL}$ and having an acceptable quality of life refused to increase their daily methadone dose, and when the increase was repeatedly recommended, they preferred to abandon the trial. They feared the previously experienced methadone dosedependent side effects, including constipation, excess sweeting, drowsiness or other sleep disorders and decreased libido. Some of them were also aware that large doses of methadone may increase the Q-T interval and increase the risk of sudden death. On the basis of these preliminary observations, the trial steering committee decided to amend the protocol and to reduce the lower limit of the therapeutic window from 100 to $80 \mathrm{ng} / \mathrm{mL}$ of plasma R-methadone concentration. The upper limit remained at $250 \mathrm{ng} / \mathrm{mL}$, but in our population, a very limited number of patients reached such levels (Fig. 3a in Mannaioni et al. [1]). Furthermore, we confirmed that plasma concentrations above $200 \mathrm{ng} / \mathrm{mL}$ were reached in patients with an oral methadone dose intake of as low as 60 or as high as $200 \mathrm{mg}$ / day (see the above-mentioned Fig. 3a). Eap et al. [3] reported that a plasma trough concentration of R-methadone of $250 \mathrm{ng} / \mathrm{mL}$ may be reached with doses as low as $55 \mathrm{mg} /$ day or as high as $921 \mathrm{mg} /$ day, thus making it particularly difficult to obtain high plasma R-methadone levels. A reasonable explanation of these data is based on the possibility that in a significant percentage of patients, large methadone doses may increase its own metabolism. It is probably worthwhile to outline that in our controlled trial, all the patients succeeding in maintaining R-methadone plasma concentration within the range of $80-250 \mathrm{ng} / \mathrm{mL}$ at 0 and 6 months continued to be benefitted by treatment for the whole duration of the trial.

Our observations were in general agreement with the data published in the mentioned Eap et al. [2, 3] papers. These

\section{KARGER}

(c) 2018 S. Karger AG, Basel

E-Mail karger@karger.com

www.karger.com/ear
Flavio Moroni and Guido Mannaioni

Department of Neurofarba, University of Firenze

Viale Pieraccini 6

IT-50134 Florence (Italy)

E-Mail flavio.moroni@unifi.it 
papers report that 86 out of 99 MMT patients had plasma R-methadone concentration in the range of $50-175 \mathrm{ng} / \mathrm{mL}$, while only 4 patients had plasma R-methadone concentration above $250 \mathrm{ng} / \mathrm{ml}$ (see the graph in Fig. 1 of Eap et al. [3]). Similar results were obtained by Meini et al. [4]. In their more recent report, at least $80 \%$ of the MMT patients had plasma Rmethadone concentration in the range $50-150 \mathrm{ng} / \mathrm{mL}$ and only 4 patients had methadone concentration above $250 \mathrm{ng} /$ $\mathrm{mL}$ (Fig. 2). Furthermore, the same paper states that the average plasma concentration of R-methadone in MMT patients not using illicit opioids was $93 \mathrm{ng} / \mathrm{mL}$, while the average $\mathrm{R}$-methadone concentration in plasma of the patients with opi- oid positive urines was $77 \mathrm{ng} / \mathrm{mL}$. We found that approximately $30 \%$ of MMT patients with plasma R-methadone concentrations in the "target" range failed to stop their use of illicit opioids. However, a significant reduction of this use took place in the course of one year of treatment. It is therefore particularly important to obtain an "appropriate" methadone dose, which is able to improve retention in treatment.

In conclusion, "maintenance in methadone treatment" and "elimination of illicit heroin use" are different goals and may require a different pattern of R-methadone plasma concentrations. The therapeutic window of $80-250 \mathrm{ng} / \mathrm{mL}$ of R-methadone was selected to test the possibility of im- proving the percentage of patients remaining in treatment possibly with an acceptable quality of life. The fear or the actual presence of side effects may prevent a significant percentage of patients to increase their daily methadone dose, thus leading to the abandonment of the therapeutic program. Finally, our data clearly suggests that the administration of an appropriate methadone dose is only one of the factors that may improve the life of patients with severe heroin use disorder.

\section{Disclosure Statement}

The authors have no conflicts of interest to disclose.

\section{References}

1 Mannaioni G, Lanzi C, Lotti M, Galli V, Totti A, Pacileo I, Sili M, Pracucci C, Dilaghi A, Bertieri L, Quaranta M, Orsini F, Occupati B, Michahelles A, Ciuti R, Bianchini E, Fabbro G, Biggeri A, Masini E, Moroni F: Methadone dose adjustments, plasma R-methadone levels and therapeutic outcome of heroin users: a randomized clinical trial. Eur Addict Res 2018;24:9-18.
Eap CB, Bourquin M, Martin J, Spagnoli J, Livoti S, Powell K, Baumann P, Deglon J: Plasma concentrations of the enantiomers of methadone and therapeutic response in methadone maintenance treatment. Drug Alcohol Depend 2000;61:47-54.

-3 Eap CB, Buclin T, Baumann P: Interindividual variability of the clinical pharmacokinetics of methadone: implications for the treatment of opioid dependence. Clin Pharmacokinet 2002;41:1153-1193.

Meini M, Moncini M, Daini L, Giarratana T, Scaramelli D, Chericoni S, Stefanelli F, Rucci P: Relationship between plasma concentrations of the l-enantiomer of methadone and response to methadone maintenance treatment. Eur J Pharmacol 2015;760: $1-6$. 